\title{
Recency, Repetition, and the Multidimensional Basis of Recognition Memory
}

\author{
- Bradley R. Buchsbaum, Sabrina Lemire-Rodger, Ashley Bondad, and Alexander Chepesiuk \\ Rotman Research Institute, Baycrest University of Toronto, Psychology, Toronto, Ontario M6A 2E1, Canada
}

Recency and repetition are two factors that have large effects on human memory performance. One way of viewing the beneficial impact of these variables on recognition memory is to assume that both factors modulate a unidimensional memory trace strength. Although previous functional neuroimaging studies have indicated that recency and repetition may modulate similar brain structures, particularly in the region of the inferior parietal cortex, there is extensive behavioral evidence that human subjects can make independent and accurate recognition memory judgments about both an item's recency and its frequency. In the present study, we used fMRI to examine patterns of brain activity during recognition memory for auditory-verbal stimuli that were parametrically and orthogonally manipulated in terms of recency and number of repetitions. We found in a continuous recognition paradigm that the lateral inferior parietal cortex, a region that has previously been associated with recollective forms of memory, is highly sensitive to recency but not repetition. In a multivariate analysis of whole-brain activation patterns, we found orthogonal components that dissociated recency and repetition variables, indicating largely independent neural bases underlying these two factors. The results demonstrate that although both recency and repetition dramatically improve recognition memory performance, the neural bases for this improvement are dissociable, and thus are difficult to explain in terms of access to a unitary memory trace.

Key words: fMRI; memory; parietal; recency; recognition; repetition

\section{Introduction}

In tests of memory, it is normally the case that items encountered more recently are recognized faster and more accurately than those encountered less recently. Likewise, items that have been presented multiple times are more easily recognized than those that have never, or only rarely, repeated. The effects of recency and repetition may both be understood as modulating an underlying quantity, memory strength, that indexes the amount of information available about a stored memory trace (Wells, 1974; Wickelgren, 1974). There is nevertheless extensive behavioral evidence that human subjects can, if required, reliably differentiate between these two variables (Peterson et al., 1969; Hintzman et al., 1972; Wells, 1974; Hintzman, 1988, 2010).

In the cognitive neuroscience of human memory, however, there is still considerable debate as to whether there exist neural structures whose activity tracks a unidimensional memory strength quantity. Because recency and repetition both have large effects on memory performance, the joint exploration of these two factors in the context of recognition memory offers a power-

\footnotetext{
Received July 21, 2014; revised Jan. 5, 2015; accepted Jan. 7, 2015

Author contributions: B.R.B. designed research; B.R.B., S.L.-R., A.B., and A.C. performed research; B.R.B., S.L.-R., A.B., and A.C. analyzed data; B.R.B. and S.L.-R. wrote the paper.

This work was supported by the Natural Sciences and Engineering Research Council of Canada, and the Canadian Institutes of Health Research.

The authors declare no competing financial interests.

Correspondence should be addressed to Dr Bradley R. Buchsbaum, Rotman Research Institute, Baycrest, 3560 Bathurst Street, Toronto, 0N M6A 2E1, Canada. E-mail: bbuchsbaum@research.baycrest.org.

DOI:10.1523/JNEUROSCI.2999-14.2015

Copyright $\odot 2015$ the authors $\quad 0270-6474 / 15 / 353544-11 \$ 15.00 / 0$
}

ful way to probe for the existence of a neural center that is generically sensitive to increases in memory strength.

Previous work has shown that recency and repetition manipulations modulate neural activity in the left inferior parietal cortex (LIPC-AG), in the vicinity of the angular and supramarginal gyri (LIPC-SMG; Yassa and Stark, 2008; Greve et al., 2010). A number of recent human neuroimaging studies have shown that activity in the angular gyrus (LIPC-AG) is sensitive to recency in memory tasks (Nee and Jonides, 2008; Buchsbaum and D’Esposito, 2009; Greve et al., 2010; Huijbers et al., 2010; Buchsbaum et al., 2011a,b). These findings seem to be consistent with functional neuroimaging studies showing enhanced LIPC-AG activation during recollective forms of long-term memory retrieval, as assessed primarily by subjective measures of conscious memory access (e.g., the remember/know paradigm; Hutchinson et al., 2009; Johnson et al., 2013).

Here we propose that the reason LIPC-AG is sensitive to both short-term recency and recollection from long-term memory is because in both cases the retrieved memory is not merely strong but is also contextually specific; that is, the reactivated memory contains perceptual, temporal, spatial, or other information that uniquely distinguishes it from other similar events. In contrast, because repetition may introduce multiple memory traces (Hintzman, 1988) that are embedded in a variety of encoding contexts (Martin, 1968; Melton, 1970; Xue et al., 2010), repetition promotes the formation of strong memories that lack contextual specificity. Thus, we propose that the LIPC-AG should be sensitive to manipulations of recency, but not necessarily repetition.

To date, however, no study has compared the effects of repetition and recency in a single functional neuroimaging study. 


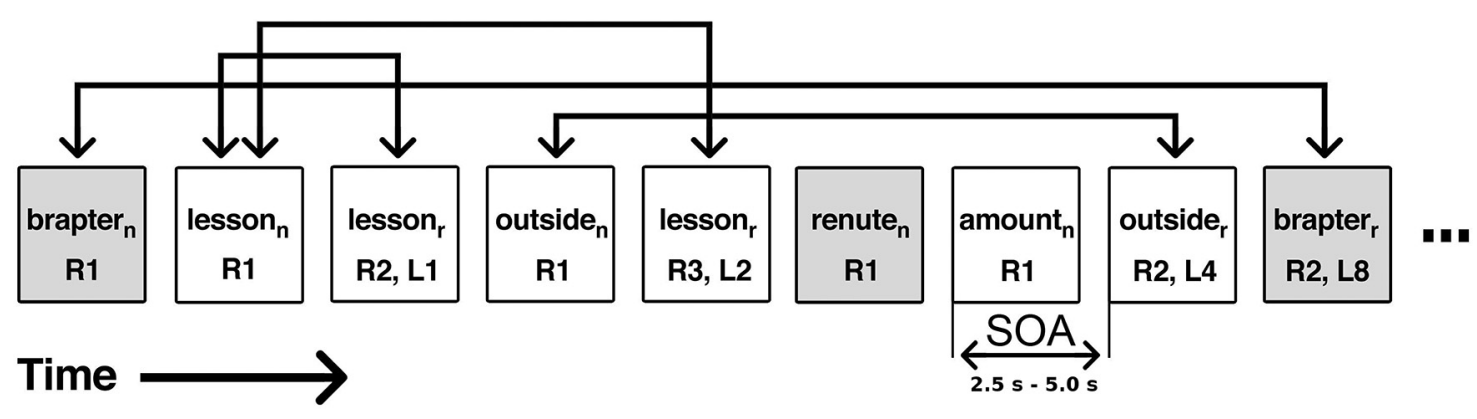

Figure 1. Schematic overview of continuous recognition task. Two-syllable words and pseudowords were auditorily presented in a continuous stream separated by a variable duration interstimulus interval (2.5-5 s). Each box represents a single auditory presentation of the depicted word, with the subscripts " $n$ " and " $r$ " indicating whether the item is novel or repeating, respectively. Below the word is the condition label, where "R1" for example means "repetition number 1" and "R2, L1" means "repetition number 2 and lag 1." The connecting lines above the boxes show the temporal relation between repeating items.

Here we factorially manipulate these two variables to test whether activation in the LIPC-AG is sensitive to the contextual specificity of a retrieved representation rather than its overall memory strength.

\section{Materials and Methods}

Participants. Twenty-three healthy subjects (14 females; age, 19-30 years, $\mathrm{M}=22.5$ years, $\mathrm{SD}=2.9$ ) gave informed consent according to procedures approved by the Research Ethics Board of the Rotman Research Institute. All were right-handed, fluent English speakers with normal hearing. All had normal or corrected-to-normal vision, and had at least 13 years of education $(\mathrm{M}=15.5$ years, $\mathrm{SD}=1.5)$. None of the subjects had a history of neurological illness. Three subjects were eliminated from statistical analyses due to poor performance (excessive rate of nonresponding; $>10 \%$ nonresponses) and an additional three subjects were eliminated due to ( $>5 \mathrm{~mm}$ maximal displacement) head motion, leaving 17 subjects ( 9 females).

Experimental stimuli. A set of 334 two-syllable nouns was selected from the MRC psycholinguistic database (Wilson, 1988). Relevant indices for the word set are the following: familiarity: mean $=524.5, \mathrm{SD}=180.4$; average Kucera-Francis written frequency: mean $=87.1, \mathrm{SD}=107.3$; imageability: mean $=429.6, \mathrm{SD}=162.5$. The initial 334 real words were then used to create pseudowords by randomly selecting pairs of words and swapping their first and second syllables to create pseudowords. For example, the words "trellis" and "below" could be used to create the pseudowords "trelow" and "bellis." Using this method, a set of 300 pseudowords was generated that preserved the bigram frequency characteristics of the real words. Six participants then rated each of the pseudowords on a scale of $1-5$ as to how similar it was to a real English word $(1=$ does not sound like a real word, $5=$ sounds very much like a real word). Pseudowords that were rated $>2$ SD above the "wordlikeness" mean were dropped from the stimulus list, leaving 276 pseudowords in the final set. All the words and pseudowords (610 in total) were then converted to audio files using online text-to-speech software Neospeech (female voice).

fMRI scanning methods. Functional and structural brain images were acquired with a 3 tesla Siemens scanner using a 32-channel Siemens head coil. All subjects were scanned over seven runs, each of which lasting 6 min and $52 \mathrm{~s}$. There was a $\sim 1$ min break between each of the seven scans during which subjects awaited the next experimental block. The total fMRI scanning time was $46 \mathrm{~min}$ and the experiment from beginning to end was $\sim 1 \mathrm{~h}$ and $30 \mathrm{~min}$.

Functional images were collected with a $\mathrm{T} 2{ }^{*}$-weighted echo-planar imaging (EPI) sequence (302 time points, $\mathrm{TR}=1370 \mathrm{~ms}$; $\mathrm{TE}=27 \mathrm{~ms}$; $\mathrm{FOV}=225 \mathrm{~mm}$; flip angle $=62^{\circ} ; 96 \times 96$ matrix). Image volumes were acquired in 24 oblique axial slices (thickness $=3.5 \mathrm{~mm}$; interslice gap $=$ $0.4 \mathrm{~mm}$; in-plane resolution $=1.88 \times 1.88 \mathrm{~mm}$ ). In addition, highresolution structural images were acquired with a T1-weighted MP-RAGE sequence in 160 oblique axial slices (thickness $=1.0 \mathrm{~mm}$; in-plane resolution $=0.975 \times 0.975 \mathrm{~mm}$ ). Wedge sponges were used to stabilize the head, minimizing head motion. The experimental paradigm was programmed and presented using PXLab software (Hans Irtel, University of Mannheim, Mannheim, Germany), which ran on a Dell laptop. Auditory stimuli were delivered via MR-compatible Avotec headphones. Subjects also wore earplugs for additional sound attenuation of the scanner background noise. Visual stimuli (instructions and fixation cross) were presented on a rear-projection screen. Participants viewed the screen via a mirror mounted inside the head coil and responded by pressing two buttons on a four-button fiber-optic response pad (Current Designs), which rested on their thigh, using their dominant (right) hand.

Experimental task. Subjects performed a continuous recognition paradigm with auditory-verbal stimuli (Fig. 1). During each of the seven scanning runs, subjects were presented with a sequence of 100 words for which they made a binary old/new recognition memory decision. The stimulus onset asynchrony for the words was distributed uniformly over the range $2.5-5 \mathrm{~s}$. For each word in the sequence, subjects pressed the right button on the response pad if the item was new (i.e., encountered for the first time in the experiment) and the left button if the item was judged to be old (i.e., previously encountered in the experiment). There were three main experimental manipulations: word type, repetition number, and lag. Word type refers to whether the item was a real twosyllable English language word or a two-syllable pseudoword. Note that the word type manipulation was not a central focus in this study, but was included to ensure that lag and repetition effects would generalize across pre-experimental familiarity with individual items. Repetition number refers to the number of times the current item had already been presented, ranging from zero (first time, e.g., "new") to six. Lag refers to the difference in the ordinal position of the current item and the last time that item was presented. For repeated items, lag could take on the following values: 1, 2, 4, 8, 16, 32 (where lag 1 is an immediate repetition). For new items, lag did not have a value. The full set of "old" trials formed a two (word type) by six (repetition) by six (lag) factorial design. The set of "new" items did not fit within this factorial structure and was treated as a separate group of trials consisting of two conditions ("new word" and "new pseudoword"). Fifty-four (27 words, 27 pseudowords) items repeated exactly six times during the experiment, and each repetition of a given word was at a different level of the lag factor. There were therefore $6 \times 54=324$ trials involving an item repetition ( $46 \%$ of all trials). The remaining 376 trials consisted of new items (half of which were real words and half pseudowords). Items were sampled randomly from the word and pseudoword stimulus pools so that not all subjects were presented with the exact same set of items.

A particular item never repeated at the same lag twice, and a repeating item occurred at all of the lag levels exactly once. The ordering of the lags for a repeating item was based on a random shuffling of the lag levels. For example, if the word "bottom" was designated as a repeating item, it would appear seven times: first as a new item and thereafter as an old item where it would occur at a different lag for each of the 6 repetitions (e.g., 2-32-1-16-4-8). Once a word had repeated six times, it would not appear again during the experiment. To avoid having more of the initial repetitions (e.g., repetitions 1 and 2) occur during the early part of the experiment and the late repetitions (e.g., repetitions 5 and 6 ) occur at the end 
Table 1. BADA analysis: locations of peak voxel loadings for PC1 (lag)

\begin{tabular}{|c|c|c|c|c|c|}
\hline$x$ & $y$ & $Z$ & Max Z-score & N voxels & Region \\
\hline \multicolumn{6}{|c|}{ Positive loadings } \\
\hline-5 & 23 & 47 & 5.13 & 111 & Cingulate gyrus \\
\hline-32 & 32 & 8 & 3.92 & 16 & Anterior insula \\
\hline \multicolumn{6}{|c|}{ Negative loadings } \\
\hline 19 & 56 & 35 & 6.37 & 371 & Frontal pole \\
\hline-38 & 29 & 47 & 5.52 & 76 & Middle frontal gyrus \\
\hline-20 & 59 & 29 & 5.08 & 47 & Frontal pole \\
\hline 7 & 50 & 14 & 4.14 & 28 & Paracingulate gyrus \\
\hline 28 & 20 & -13 & 4.51 & 21 & Frontal orbital cortex \\
\hline 25 & 26 & 53 & 3.53 & 19 & Middle frontal gyrus \\
\hline 28 & 8 & 50 & 3.61 & 19 & Middle frontal gyrus \\
\hline 49 & -43 & 29 & 9.44 & 2634 & $\begin{array}{l}\text { Angular gyrus/superior temporal } \\
\text { sulcus }\end{array}$ \\
\hline-59 & -49 & 41 & 9.73 & 811 & Supramarginal gyrus \\
\hline 1 & -43 & 41 & 4.78 & 146 & Precuneous cortex \\
\hline-68 & -25 & -4 & 5.48 & 104 & Middle temporal gyrus, posterior \\
\hline-53 & 2 & -34 & 4.50 & 31 & Middle temporal gyrus, anterior \\
\hline
\end{tabular}

experiment, "repeating" words were introduced periodically throughout the experiment. This was done in such a way as to minimize the relation between the absolute serial position of items and repetition number. This scheme produced an average correlation over all stimulus randomizations between trial number (1-700) and repetition number (1-6) was low (Spearman rank correlation: 0.08$)$. As a consequence of this scheme, however, a small proportion $(4 \% ; 28 / 700)$ of repetition trials crossed over scanning run boundaries. Because this affected the absolute time between these repetitions on these trials (but not the ordinal lag), they were eliminated from all behavioral and fMRI analyses. Most of these "cross-run" trials (75\%) were of lag 16 and 32 and the remaining $25 \%$ were from lags 1, 2, 4, and 8 . Because these cross-run trials were removed from all analyses the net effect was that there were somewhat fewer remaining analyzable observations at longer lags (e.g., 54 trials at lag $1 ; 40$ trials at lag 32).

Finally, it should be noted that although this experiment has a large number of nominal "conditions," we used statistical analyses that generally treated both lag and repetition as continuous variables. Thus, we were primarily interested in monotonic relationships between keydependent variables (BOLD, RT, accuracy) and the integer-valued independent variables, lag and repetition. To take advantage of the parametric nature of our design and to maximize power, we have avoided categorical (e.g., ANOVA-based) analyses in favor of analyses in which lag and repetition are entered as continuous measures.

fMRI data processing and statistical analysis. For each scanning run, 302 EPI images were obtained in DICOM format and exported using AFNI (Cox, 1996) into NIFTI-1 format. For each subject, images were motioncorrected and realigned to the mean image volume for the first run of the session using the AFNI program 3dvolreg. Functional images were then smoothed with a $5 \mathrm{~mm}$ full-width at half-maximum Gaussian kernel. All statistical analyses were performed on these smoothed and realigned images.

Each subject's high-resolution anatomical MP-RAGE scan was normalized to Montreal Neurological Institute (MNI) stereotaxic space with a nonlinear transformation using nonlinear symmetric normalization as implemented in ANTS (Avants et al., 2008). An additional six-parameter rigid-body registration between each subject's mean functional image was performed to derive a transformation between each subject's native EPI space and the subject's high resolution anatomical image. For display purposes, data from group analyses were projected on to a standard MNI surface template (the fsaverage cortical surface from FreeSurfer; Dale et al., 1999) using the AFNI program $3 d$ Vol2Surf and visualized using the AFNI program SUMA.

Univariate statistical analyses of $\mathrm{ARI}$ data. Single-subject analyses using the general linear model (GLM) were performed using the AFNI program $3 d$ Deconvolve. To increase the number of observations within each cell and thus improve the precision of condition-wise parameter estimates, we collapsed across repetitions 1-2, 3-4, and 5-6, respectively.
Table 2. BADA analysis: locations of peak voxel loadings for PC2 (repetition)

\begin{tabular}{lrrlrl}
\hline$x$ & $y$ & $z$ & Max Z-score & Size & Region \\
\hline Positive loadings & & & & & \\
-2 & 17 & -1 & 4.85 & 59 & Paracingulate gyrus \\
-2 & 38 & -19 & 3.74 & 18 & Frontal medial cortex \\
-11 & 53 & 2 & 4.09 & 16 & Paracingulate gyrus \\
64 & -28 & 32 & 4.49 & 45 & Supramarginal gyrus \\
Negative loadings & & & & & \\
-44 & 5 & 35 & 5.35 & 282 & Middle frontal gyrus \\
1 & 26 & 53 & 5.80 & 210 & Superior frontal gyrus \\
-32 & 26 & -1 & 6.19 & 129 & Insular cortex \\
37 & 23 & -4 & 5.31 & 86 & Insular cortex \\
-44 & 53 & 2 & 4.82 & 68 & Frontal pole \\
-68 & -31 & 2 & 4.20 & 25 & Middle temporal gyrus \\
-44 & -49 & 50 & 4.74 & 130 & Lateral occipital cortex \\
-14 & 5 & 14 & 3.81 & 16 & Thalamus \\
\hline
\end{tabular}

This yielded a regression model with 36 total conditions $(6$ lag $\times 3$ repetition $\times 2$ word type).

Each of the 36 conditions was modeled by convolving a hemodynamic response function (SPM canonical function as implemented in AFNI) with the onset and duration of the experimental events. An additional set of five nuisance regressors (a constant term plus linear, quadratic, and higher-order polynomial terms) was included for each scanning run to model low-frequency noise in the time series data. Trials in which subjects failed to respond, responded too quickly $(<500 \mathrm{~ms})$, or took too long to respond ( $>3000 \mathrm{~ms}$ ) were excluded from the main analysis and modeled with a separate "error" regressor (between $4 \%$ and $6 \%$ of all trials over subjects). All of the remaining trials were included, both correct and incorrect. The rationale for including all trials, rather than only correct trials, is because of our primary interest was in tracking the relationship between independently manipulated variables (lag and repetition) and their relationship to behavioral and neural indices of memory strength. Thus, if we were to only include correct trials, we would be effectively examining trials for which memory strength was by definition high, thus distorting our estimate of the relationship between the independent variables of interest and memory strength; moreover, this distortion would be disproportionate in the more difficult conditions, i.e., those with longer lags and/or longer lags and a lower number of repetitions.

Statistical contrasts at the single subject level were computed as weighted sums of the estimated $\beta$ coefficients divided by an estimate of the SE, yielding a $t$ statistic for each voxel in the image volume.

A priori ROI analysis. To examine activation in the portion of the LIPC-AG that has previously been shown to be recency-sensitive, we performed an ROI analysis. To avoid a selection bias in defining the area of interest, we used MNI coordinates reported in a number of previous functional neuroimaging studies showing recency effects in the LIPC-AG (Buchsbaum and D'Esposito, 2009; Greve et al., 2010; Huijbers et al., 2010; Kimura et al., 2010; Oztekin et al., 2010; Buchsbaum et al., 2011a; Nee and Jonides, 2011) to define a priori ROIs for the left and right hemispheres. This was done by taking the mean of all the coordinates falling in the LIPC-AG from these studies and defining a spherical ROI with an $8 \mathrm{~mm}$ radius around the coordinate centroid (left LIPC-AG: $x=$ $-52, y=-57, z=39$; right LIPC-AG: $x=53, y=-50, z=30)$. Left and right ROI masks were then used to extract averaged values from spatially normalized $\beta$ images estimated in subject-specific GLM analyses.

Multivariate statistical analysis. To examine distributed patterns of activation in the brain associated with experimental conditions we conducted barycentric discriminate analysis (BADA) (Abdi, 2010) on the set of spatially normalized $\beta$ estimates from the single-subject GLM analyses as an input data matrix. BADA is a multivariate statistical method that decomposes a data matrix into orthogonal factors that capture the association between a set of $J$ variables (voxels) and $N$ categories (conditions; Abdi, 2010; Abdi et al., 2012; Buchsbaum et al., 2012). Each observation is represented by the 1 by $J$ vector of the activation values of its voxels. Then each category of interest is represented by the barycenter of its 
Table 3. Peak activations for correlations with lag, repetition, and memstrength

\begin{tabular}{|c|c|c|c|c|}
\hline$x$ & $y$ & $z$ & $N$ voxels & Region \\
\hline \multicolumn{5}{|c|}{ Positive correlations } \\
\hline \multicolumn{5}{|l|}{ Best Lag } \\
\hline 13 & 65 & 11 & 261 & Frontal pole \\
\hline-38 & 26 & 38 & 232 & Middle frontal gyrus \\
\hline-23 & 65 & 20 & 41 & Frontal pole \\
\hline 25 & 8 & 47 & 30 & Middle frontal gyrus \\
\hline 37 & 62 & -10 & 27 & Frontal pole \\
\hline 31 & 20 & -19 & 23 & Frontal orbital cortex \\
\hline 46 & 11 & -43 & 2701 & Angular gyrus \\
\hline-56 & -49 & 5 & 764 & Supramarginal gyrus \\
\hline 1 & -52 & 32 & 94 & Precuneous cortex \\
\hline-71 & -25 & -16 & 75 & Middle temporal gyrus \\
\hline-53 & -4 & -40 & 24 & Inferior temporal gyrus \\
\hline \multicolumn{5}{|l|}{ Best Rep } \\
\hline 1 & 38 & -10 & 45 & Paracingulate gyrus \\
\hline 58 & -28 & 26 & 19 & Supramarginal gyrus \\
\hline \multicolumn{5}{|c|}{ Best memstrength } \\
\hline 22 & 14 & 47 & 81 & Middle frontal gyrus \\
\hline 4 & 56 & 26 & 72 & Frontal pole \\
\hline-29 & 26 & 44 & 40 & Middle frontal gyrus \\
\hline 7 & 56 & 5 & 31 & Frontal pole \\
\hline-23 & 44 & 44 & 31 & Frontal pole \\
\hline-65 & -46 & 26 & 55 & Supramarginal gyrus \\
\hline 64 & -31 & 29 & 22 & Supramarginal gyrus \\
\hline 43 & -58 & 17 & 127 & Lateral occipital cortex \\
\hline-32 & -88 & 29 & 19 & Lateral occipital cortex \\
\hline-32 & -7 & -19 & 16 & Amygdala \\
\hline \multicolumn{5}{|c|}{ Negative correlations } \\
\hline \multicolumn{5}{|l|}{ Best Lag } \\
\hline 28 & -61 & -1 & 17 & Precuneous cortex \\
\hline 19 & -100 & 11 & 37 & Occipital pole \\
\hline \multicolumn{5}{|l|}{ Best Rep } \\
\hline-47 & 20 & 14 & 228 & Middle frontal gyrus \\
\hline-50 & 20 & -13 & 98 & Insular cortex \\
\hline-8 & 38 & 35 & 95 & Superior frontal gyrus \\
\hline 58 & 23 & 17 & 77 & Middle frontal gyrus \\
\hline 40 & 23 & -10 & 57 & Frontal orbital cortex \\
\hline-41 & 50 & -4 & 54 & Frontal pole \\
\hline 52 & -16 & -4 & 110 & Superior temporal gyrus \\
\hline-65 & -28 & -4 & 78 & Superior temporal gyrus \\
\hline-59 & 2 & -10 & 61 & Superior temporal gyrus \\
\hline 49 & 11 & -13 & 27 & Temporal pole \\
\hline-35 & -49 & 35 & 123 & Lateral occipital cortex \\
\hline \multicolumn{5}{|c|}{ Best memstrength } \\
\hline 13 & 35 & 23 & 225 & Paracingulate gyrus \\
\hline-50 & 20 & 20 & 96 & Middle frontal gyrus \\
\hline-32 & 29 & -1 & 91 & Insular cortex \\
\hline 34 & 17 & -4 & 45 & Insular cortex \\
\hline-17 & 20 & -22 & 18 & Frontal orbital cortex \\
\hline
\end{tabular}

observations (i.e., the weighted mean; the barycenter is also called the center of gravity of the observations of a given category). A generalized principal component analysis is finally performed on this category by variable matrix. In the present case, the "observations" consist of the $\beta$ image maps for each of the 36 conditions in the experiment. The goal of $\mathrm{BADA}$ is to discover reliable multivariate patterns of activation that best explain the total variance in the set of activation images.

BADA gives a set of discriminant factor scores for the categories and a set of loadings for the voxels. First, the number of significant components is assessed by comparing the magnitude of the eigenvalues of the solution to an empirical null distribution derived from 500 random permutations of the data matrix, which is a standard number for multivariate analyses of brain data (McIntosh and Lobaugh, 2004). Statistical significance for the BADA components is determined using 500 bootstrap replications of the original data matrix yielding pseudo Z-scores for both the factor scores and voxel loadings (McIntosh and Lobaugh, 2004). Contrast vectors encoding hypotheses of interest are correlated with the BADAderived factor scores to assess the extent to which significant components derived from the BADA solution reflect one or another aspect of the experimental design. By computing the bootstrapped correlation between a linear contrast vector parameterized by lag or repetition, we can formally assess the association between the experimental manipulations and the factors derived from the data-driven BADA solution. This allows us to test whether the most important dimensions of the data in terms of explained variance are related to the dimensions that comprise the factorial structure of the experimental design (i.e., lag and repetition). Because BADA is based on PCA, one assumption of the technique is that the data can be adequately decomposed into linear combinations of orthogonal factors, which may not be the case for all datasets.

Voxelwise analyses of lag, repetition, and memory strength. Although the BADA is very sensitive to large-scale multivariate patterns in the data, it may not be as sensitive to local activation patterns that are not part of a correlated network. To derive an index of memory strength from behavioral performance measures, we used a combination of RT and accuracy data. Combining these measures made use of both speed and accuracy data and because accuracy was near ceiling for lag 1 at all repetition levels, the RT information provides additional information to optimally sort the conditions in terms of memory strength.

Thus, we computed the rank order of mean RT over all conditions (ranking each condition from lowest to highest) and accuracy (ranking each condition from highest to lowest). To combine these two measures, we first averaged these ranks together and then reranked these averages, yielding a final nonparametric index of memory strength that could be associated with each experimental condition (henceforth, "memstrength") and was based on both RT and accuracy measures.

We then sought to identify regions in the brain whose activity profile was better predicted by memstrength than either lag or repetition alone. To achieve this we computed a voxelwise Spearman rank correlation between each of the three variables (lag, repetition, and memstrength) and each subject's set of $36 \beta$ images ( 6 lag $\times 3$ repetition $\times 2$ word type). At the group level, we then compared the three sets of within-subject correlations for every voxel in MNI space to one another using paired $t$ tests on the Fisher $Z$-transformed correlations. To identify voxels in the brain that were best modeled by one of the three variables we used two criteria. First, to classify one of the variables as the "nominally best model" for a voxel we required that it show a significant relationship with the BOLD activity estimate $(p<0.005$, cluster extent $>18$ voxels as determined by Monte Carlo simulation using AFNI program 3 dAlphaSim) and have a numerically larger mean correlation than both of the other two measures. Second, to classify a variable as the "reliably best model" we required that it show a significant correlation with the fMRI signal on its own $(p<0.005$, cluster extent $>18$ voxels $)$ and have a significantly larger $(p<0.005)$ average correlation than both of the other two measures. This latter requirement was quite stringent because our performance-based memory strength variable was necessarily correlated with both lag and repetition independent variables. For that reason we report results for three thresholds (nominal best model, reliably best at $p<0.05$, and reliably best at $p<0.005$; see Table 3 and Fig. 7).

\section{Results}

\section{Behavioral results}

Before analyzing RT and accuracy, we first removed all trials for which RT was $<500 \mathrm{~ms},>3000 \mathrm{~ms}$, or trials in which a repeating item crossed a scanning run boundary. We then performed a linear mixed effects analysis with three independent variables: word type (real, pseudo) and repetition number (1-6), and log lag $(1,2,4,8,16,32)$ as continuous variables, plus all higherorder linear interactions. The "lag" variable was log-transformed so that its relation to RT was more linear. This basic linear model accounted for $84 \%$ of the variance of the group means. As recommended by Pinheiro and Bates (2000), we used bootstrap resampling to assess statistical significance of the fixed effects terms in the model. We performed 1000 bootstrap iterations, sampling 
with replacement from subjects. Pseudo $Z$-scores were then computed by dividing the model coefficients by the SD of the estimates derived from the bootstrap resampling procedure. Subjects were faster to respond to real (mean $\mathrm{RT}=1064 \mathrm{~ms}$ ) than to pseudowords (mean RT $=1100$ $\mathrm{ms})$ and this effect was statistically significant $(Z=-4.73, p<0.0001)$. Significant linear effects of repetition number $(Z=$ -11.84, $p<0.0001)$ and $\log \operatorname{lag}(Z=$ 17.63, $p<0.0001$ ) were also observed. There were also significant interactions between the linear effect of repetition number and $\log \operatorname{lag}(Z=-2.39, p=$ $0.016)$, word type and $\log \operatorname{lag}(Z=-2.72$, $p=0.006)$, and word type and repetition number $(Z=2.17, p=0.03)$.

We also assessed the percentage of variance explained by lag and repetition variables, computed separately for each subject. The lag and repetition variables accounted for an average of $16 \%$ (SD = $0.08)$ and $7 \%(\mathrm{SD}=0.04)$ of the RT variance, respectively. The average spearman rank correlation between lag and RT was $0.43(\mathrm{SD}=0.11)$ and $-0.26(\mathrm{SD}=0.08)$ between repetition and $\mathrm{RT}$. Thus, although both lag and repetition had reliable effects on RT, the former explained somewhat more total variance. Finally, we tested whether a more complex model with additional quadratic terms for both lag and repetition would yield a better fit to the data. Indeed, adding squared terms for repetition number and log lag, plus all higher-order interactions, yielded a better model fit as evaluated with a likelihood ratio test $(p<0.001)$. However, compared with the fully linear model the augmented model explained only an additional $6 \%$ (from $84 \%$ to $90 \%$ ) of the variance in fits to the group means. The cell means for all RTs with model fits from the augmented model are shown in Figure 2.

We also examined the accuracy data using a mixed effects logistic regression model with resampling to assess statistical reliability of model terms. This analysis revealed significant effects of $\log \operatorname{lag}(Z=$ $-5.41, p<0.0001)$ and repetition number $(Z=4.49, p<0.0001)$ and a nonsignificant effect word type (words more accurate than pseudowords; $Z=1.45, p=0.14$ ); that is, however, in the same direction as the corresponding RT effect. In contrast to the RT data, none of the interaction terms reached statistical significance. Thus, as expected, accuracy decreased as a function of lag and increased as a function of repetition. Overall, collapsing across repetition and word type, accuracy was near ceiling at 0.98 (proportion correct) for lag 1 and decreased monotonically to 0.85 accuracy for lag 32 . Likewise, collapsing over lag and word type, accuracy was at 0.98 for the sixth repetition and decreased monotonically to $86 \%$ for the first repetition.

\section{fMRI data analysis}

ROI analysis: $L I P C-A G$

To examine the effects of lag and repetition in the LIPC-AG, we used left and right hemisphere $8 \mathrm{~mm}$ spherical ROIs defined using MNI coordinates (see Materials and Methods) derived from previous studies that have identified recency effects in the parietal lobe (left LIPC-AG: $x=-52, y=-57, z=39$; right LIPC-AG: $x=53, y=-50, z=30)$. These ROIs were then used to extract parameter estimates from the GLM analyses conducted at the single subject level. As with the analysis of behavioral RT data, linear mixed effects models with bootstrap resampling were then performed separately for left and right hemisphere ROIs to test for statistical significance of the following model terms: log lag, repetition, word type and all higher- order interactions. As with behavioral analyses, both log lag and repetition were modeled as continuous variables rather than as unordered factors. In both hemispheres there was a significant linear effect of log lag (left: $Z=-6.15, p<0.0001$; right: $Z=-4.16, p<0.0001$ ). On the left side there was a significant log lag by repetition $(Z=$ $3.009, p=0.0043$ ) interaction, and a trend for the same interaction on the right $(Z=1.55, p=0.12)$. No other effects were statistically significant in either hemisphere. To further characterize the lag by repetition interaction, we first averaged across word type and hemisphere because both hemispheres showed a similar interaction pattern. We then examined linear repetition effects within each level of lag $(1,2,4,8,16,32)$ with a bootstrapped linear mixed model. These follow-up analyses showed that there was no linear repetition effect for lags $1,2,4$, and 8 , but there was a significant effect at lag $16(Z=3.01, p=0.004)$ and a smaller and nonsignificant lag $32(Z=1.49 ; p=0.13)$. Thus, for the longest two lags there is a modest increase in activation as a 


\section{A}

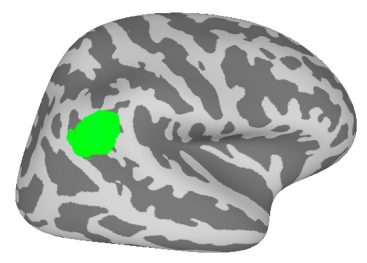

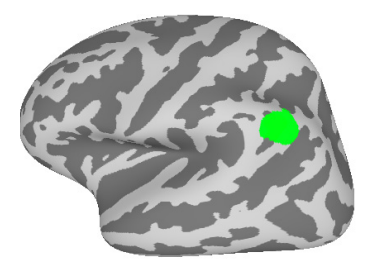

B

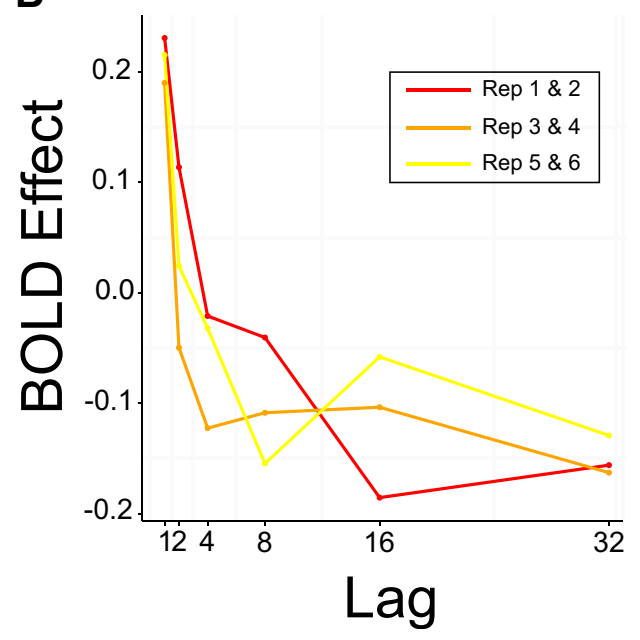

Figure 3. BOLD Activation as a function of lag and repetition in LIPC-AG ROI. $\boldsymbol{A}$, ROIs for right and left hemispheres based on average coordinates from studies in the literature that have shown recency effects in the lateral parietal cortex. $\boldsymbol{B}$, Plot of activation as a function of lag (horizontal axis) and repetition (red line: repetition 1 and 2; orange line: repetition 3 and 4; yellow line: repetition 5 and 6) averaged across word type and ROI hemisphere. Rep, Repetition number.

function of repetition, whereas for the earlier lags this effect is absent. The location of the ROIs and the pattern of effects for lag and repetition, averaged across word type and hemisphere, are presented in Figure 3, which clearly shows an activity profile that is dominated by the lag variable.

\section{Multivariate analysis of $\mathrm{fMRI}$ data}

To examine whether repetition and lag are dissociable in terms of their distributed patterns of activation, we performed BADA on the set of 36 ( 6 lag $\times 3$ repetition $\times 2$ word type $)$ spatially normalized $\beta$ images. Permutation testing with 500 randomizations showed that only the first two principal components (PC1 and PC2) were significant at the $0.05 \alpha$ level. To test for an association between the two significant components and the experimental design, we computed separate Spearman rank correlations between both lag and repetition and the two significant principal components. Statistical significance was assessed using bootstrap resampling (500 resampling iterations; McIntosh and Lobaugh, 2004) from the original data matrix, stratified by subject. This yielded a distribution of correlations from which bootstrap ratios were computed. PC1 was highly correlated with lag (Spearman rank correlation $=0.83, Z=21.6, p<0.0001$ ), but not significantly correlated with repetition (Spearman rank correlation $=$ -0.06). PC2 was significantly correlated with repetition (Spearman rank correlation $=0.82$, pseudo- $Z=23.07, p<0.0001$ ) but not with lag (Spearman rank correlation $=-0.15$ ). Thus, as can be seen in Figure 4, PC1 was strongly associated with lag and PC2 was strongly associated with repetition. The associated voxel loadings for the two significant components are shown in Figure 5 , revealing activation in the LIPC-AG extending in to the posterior temporal cortex that was positively correlated with $\mathrm{PC} 1$; and negative loadings in the anterior insula and middle frontal gyrus (for a full listing of areas, see Table 1). PC2 was associated with strong negative loadings in the anterior insula, dorsolateral prefrontal cortex, and superior temporal cortex, indicating decreased activation with increasing repetition (i.e., repetition suppression). Positive loadings for PC2 were observed in the supramarginal gyrus bilaterally and in the medial frontal cortex (Table 2). The overall dissociation between components associated with lag and repetition in the multivariate structure of the data, suggests that the two manipulations have their effect on largely independent neural processes.

Whole-brain search for a region tracking "memory strength" Although the foregoing multivariate analysis showed that lag and repetition effects are captured by orthogonal components in the full data matrix, it is nevertheless possible that activity in local areas show some reliable sensitivity to both variables, and might therefore be more closely associated with a general measure of memory strength than either lag or repetition. To derive an index of memory strength from behavioral performance measures, we used a combination of RT and accuracy data as described in Materials and Methods. The ordering of the conditions is visualized in Figure 6, where one can see a very regular ordering of memstrength across conditions such that the condition with the highest memstrength is at lag 1 and repetition 6 (Fig. 6, largest circle) and the condition with the lowest memstrength is lag 32 repetition 1 (Fig. 6, smallest circle).

We then sought to identify regions in the brain whose activity profile was better predicted by memstrength than either lag or repetition alone. To achieve this we computed a voxelwise Spearman rank correlation between each of the three variables (lag, repetition, and memstrength) and each subject's set of $36 \beta$ images ( 6 lag $\times 3$ repetition $\times 2$ word type). At the group level, we then compared the three sets of within-subject correlations for every voxel in MNI space to one another using paired $t$ tests on the Fisher $Z$-transformed correlations. To identify voxels in the brain that were best modeled by one of the three variables we used two criteria. First, to classify one of the variables as the nominally best model for a voxel we required that it should show a significant relationship with the BOLD activity estimate $(p<0.005$, cluster extent $>18$ voxels as determined by Monte Carlo simulation using AFNI program $3 d$ AlphaSim) and have a numerically larger mean correlation than both of the other two measures. Second, to classify a variable as the reliably best model we required that it be significantly correlated with the fMRI signal on its own $(p<$ 0.005 , cluster extent $>18$ voxels) and have a significantly larger $(p<0.005)$ average correlation than both of the other two measures. This latter requirement was quite stringent because our performance-based memory strength variable was necessarily 
correlated with both lag and repetition independent variables. For that reason we report results for three thresholds (nominal best model, reliably best at $p<0.05$, and reliably best at $p<0.005$ ) in Table 3 and Figure 7.

The results show that the largest positive correlations were observed with the lag variable in the LIPC-AG, bilaterally. This relationship held even for the strictest significance cutoff. Negative repetition effects (decreasing activity with increasing repetition) were also observed in the inferior and middle frontal gyrus, left intraparietal sulcus, and left and right superior temporal cortices. These effects reflect repetition suppression and were observed in temporal lobe sites that replicate auditoryverbal repetition effects shown in previous research (Buchsbaum and D'Esposito, 2009). A positive repetition effect was also seen (nominal best model) in the left SMG (anterior to the positive lag effect), consistent with the map of principal component loadings for PC2 in the BADA analysis (Fig. 5, PC2, orange colors). The anterior insula and anterior cingulate cortex bilaterally were more strongly (negatively) correlated with memstrength than either lag or repetition, whereas a positive relation was observed (nominal best model) in the SMG bilaterally and the bilateral occipital cortex on the left.

Finally, we reran the correlation analysis for the repetition variable using $\beta$ estimates from all six repetition conditions (rather than collapsing across neighboring values, e.g., 1-2, 3-4, 5-6) to confirm that our results were not distorted by averaging over conditions. We found that this analysis produced very similar results and that correlation between the collapsed and uncollapsed group statistical maps were highly correlated across voxels (Pearson correlation $=0.85$ ).

\section{Discussion}

The aim of the present study was to investigate whether there is a common neural substrate that captures variation in memory strength driven by both recency and repetition. We confirmed the strong behavioral impact of recency and repetition on recognition performance: both variables had large effects on both accuracy and RT. In particular, as the number of repetitions increased and lag decreased, subjects' behavioral performance improved. Although both of these measures had strong effects on behavioral performance, they had qualitatively different effects on patterns of brain activation. We showed, using an a priori defined ROI analysis, that activation in the portion of the LIPC-AG previously associated with recency, was here
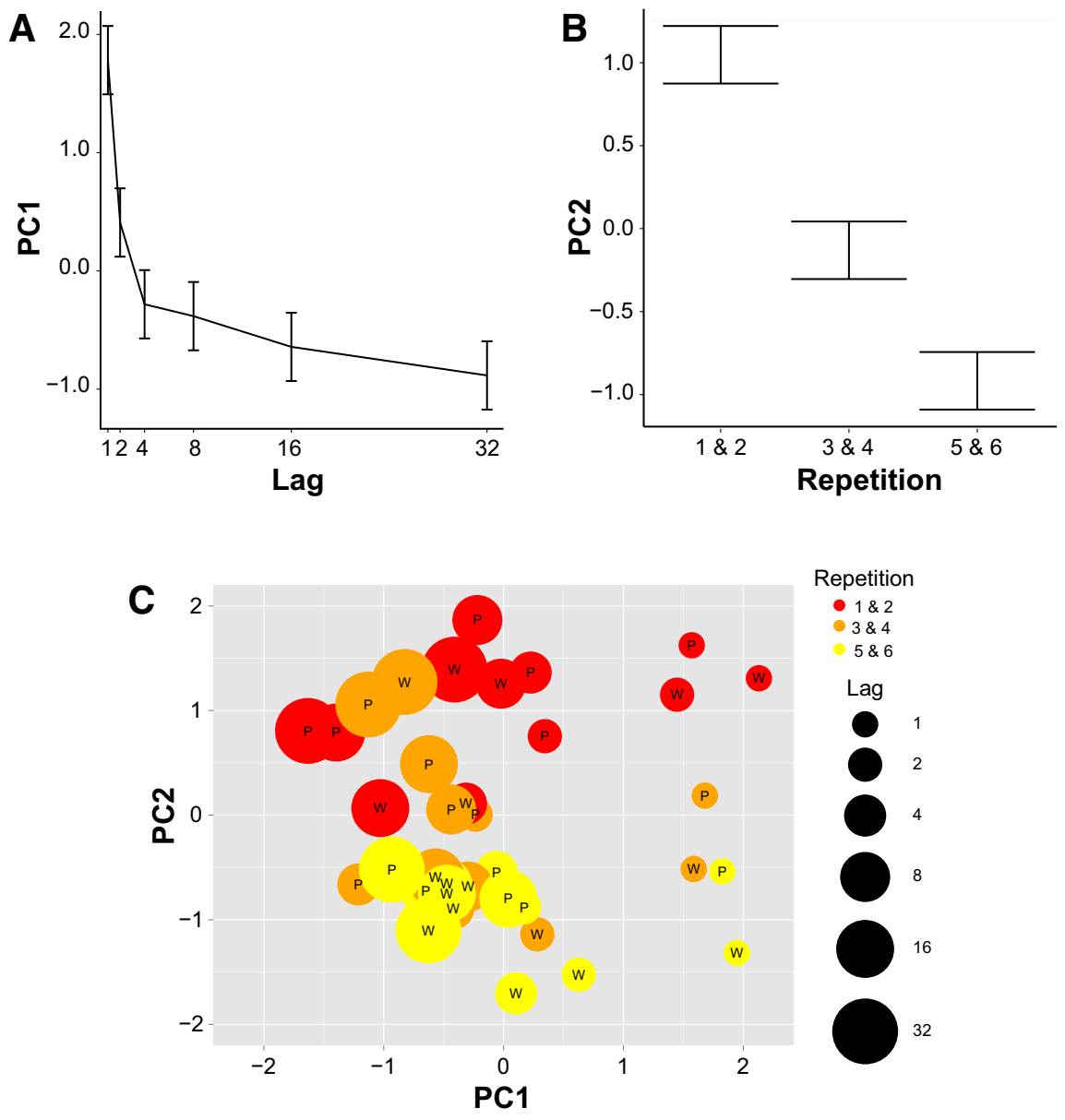

Figure 4. Plot of BADA principal components and experimental variables. $A$, Top, left, Lag is plotted on horizontal axis and the first principal component from the BADA analysis, averaged over repetition is on vertical axis. $\boldsymbol{B}$, Top, Right, Repetition plotted on horizontal axis and second principal component, averaged over lag plotted on vertical axis. C, Composite plot of first two principal components (horizontal and vertical axes), lag (indicated by radius of circles), repetition (indicated by color of circles), and word type (indicated by label inside circles).

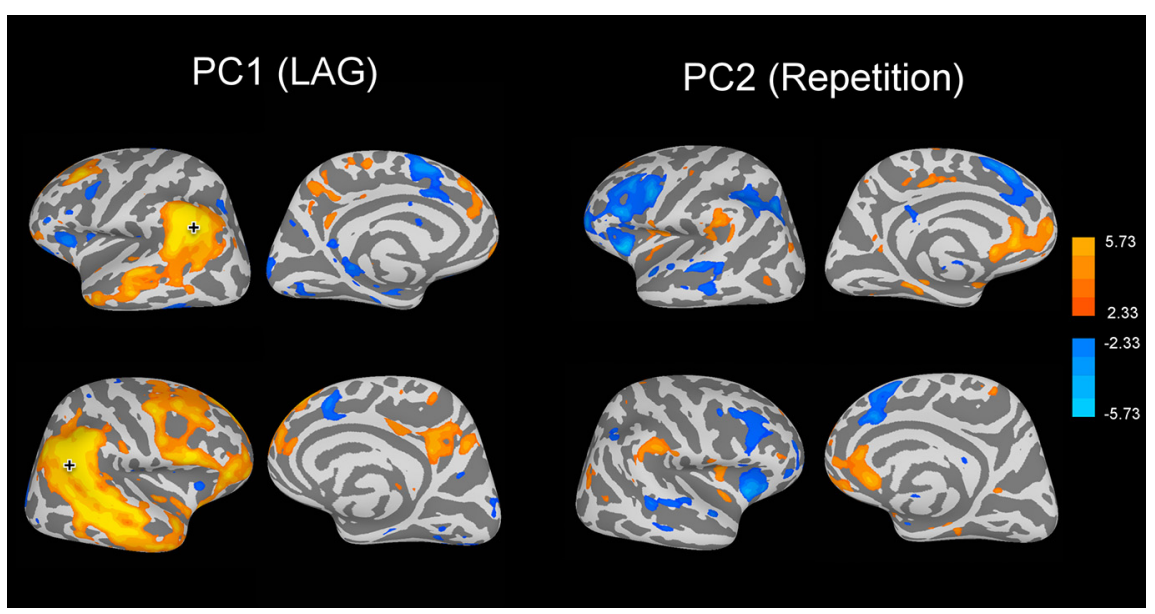

Figure 5. Voxel loadings for first two principal components of BADA analysis. Left, Top and Bottom, Lateral and medial surface views of voxel loadings for principal component 1. Right, Top and Bottom, Lateral and medial surface views of voxel loadings for principal component 2. For reference, the crosshairs on the leftmost top and bottom surfaces indicate the location of the center of the LIPC-AG ROIs shown in Figure3.

largely due to the lag variable, although there was a significant effect of repetition at lag of 16 .

Multivariate analysis of the set of condition-wise activation images with BADA revealed two significant orthogonal compo- 


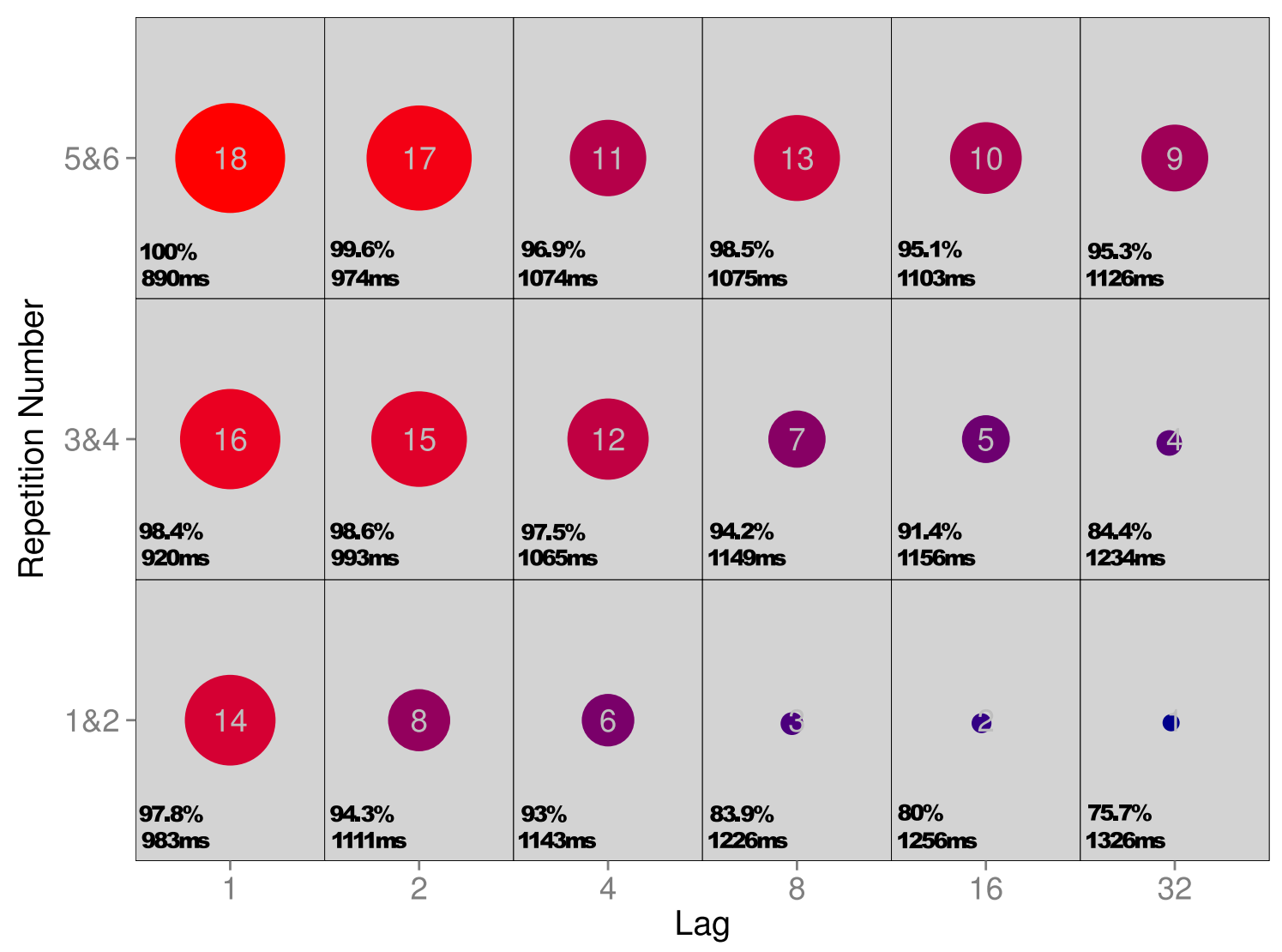

Figure 6. Plot of ranked memory strength variable for all factorial combinations of lag and repetitions. Plot shows the behaviorally derived memstrength variable that is based on the averaged rank order of RT accuracy measures for all combinations of lag and repetition. Larger circles indicate higher memory strength, with the largest circle at lag of 1 and repetition 5 and 6 (top left circle of plot) and the smallest circle at lag 32 and repetition 1 and 2 (bottom right circle in plot). For each condition, average accuracy (as percentage correct) and RT (in milliseconds) are displayed in the bottom left of the cell.

nents. The first of these components was highly correlated with lag (but not repetition), and the second was highly correlated with repetition (but not lag). This finding accords well with behavioral research that has shown that human subjects can make independent judgments about the frequency and recency of a previously encountered item (Hintzman, 2010). The clear neural dissociation between recency and repetition adds to this behavioral evidence by showing that these two factors have qualitatively distinct effects on distributed brain activity, rather than jointly modulating activity in a single neural region or network.

Finally, we examined whether a measure of memory strength, derived by rank-ordering mean behavioral performance across all combinations of recency and lag conditions, was more strongly correlated with voxelwise neural activity than with simple rank orderings determined by the independent variables lag and repetition. We found that, with the exception of the anterior cingulate gyrus and anterior insula, regions associated with cognitive control and decision-making (Dosenbach et al., 2008; Menon and Uddin, 2010), task-active brain areas were generally more sensitive to the independent variables lag or repetition than to the performance-derived composite measure of memory strength. Thus, the frequently invoked concept of memory strength may have little explanatory value when used generically, that is, without reference to the particular experimental variables, such as lag and repetition, that produce a change in memory performance. We did observe some evidence for preferential sensitivity (at the nominal significance level) to memory strength in the SMG (Fig. 6, bottom row), near a region that has previously been associated with the level of confidence in recognition memory (Moritz et al., 2006; Kim and Cabeza, 2009). However, the strength of the correlation, although numerically greater, was not reliably different from that of repetition or lag, and therefore must be treated with caution.

\section{Implications for the role of the parietal lobe in memory retrieval}

There is now a great deal of converging evidence from functional neuroimaging research implicating the parts of the parietal lobe in key components of memory retrieval. Indeed, understanding the role of the parietal cortex in memory has become a pressing matter in cognitive neuroscience research because of its seemingly disproportionate importance in functional neuroimaging studies compared with evidence from studies of patients with lesions to the area (Berryhill et al., 2007; Simons et al., 2008; Olson and Berryhill, 2009; Ciaramelli et al., 2010; Berryhill, 2012; Hower et al., 2014). To date, most of the neuroimaging work has focused on the relation between brain activity and behavioral performance, or introspective judgments about the quality of a retrieved (long-term) memory (e.g., hits vs misses, source memory attribution, remember/know judgments). It has been pointed out, however, that reliance on subjective decisions the strength or quality of retrieved memories has the drawback that they are measured rather than manipulated variables (de Zubicaray et al., 2011), and thus are susceptible to confounding factors, such as item effects. Nevertheless, current empirical consensus is that the LIPC-AG is most active when subjects are able to retrieve contex- 
tual details about the encoded event (Henson et al., 1999; Kahn et al., 2004; Wheeler and Buckner, 2004; Wagner et al., 2005; Yonelinas et al., 2005; Rissman et al., 2010; Levy, 2012; Kuhl and Chun, 2014). In contrast, a more dorsally situated region in the intraparietal sulcus of the parietal lobe has been shown to covary with increasing familiarity ratings in the absence of recollection (e.g., Vilberg and Rugg, 2007).

As mentioned in the Introduction, however, we and others (Nee and Jonides, 2008; Greve et al., 2010; Buchsbaum et al., 2011a) have shown that the LIPC-AG is highly sensitive to recency, especially when the retrieved item is within the "focus of attention" (McElree and Dosher, 1989; Cowan, 2001), for example, in the lag- 1 condition of the present study. Thus, any successful explanation of memoryrelated activity should not restrict its scope to long-term memory retrieval. Rather, one should look for a common explanation for the heightened activation associated with recency and successful recollection, as well as evidence from other cognitive domains. One potential interpretation of the common site of activation associated with (short-term) recency and (long-term) recollection is that, indeed, retrieval of a recent item is more likely to involve recollection than retrieval of a less recent item. According to this view, recency effects and recollection effects as obtained in remember/know and related paradigms are manifestations of the same underlying process (Göthe and Oberauer, 2008). Considered along these lines, LIPC-AG sensitivity to lag may be a kind of "recollection effect" that happens to occur at very short delays.

This explanation is complicated, however, by the general lack of effect of repetition on LIPC-AG activation. For instance, it has been shown that repetition of musical pieces (Gardiner et al., 1996) and words (Jones and Jacoby, 2001) increase the likelihood that an item is endorsed as "remembered" in a remember/know paradigm. However, it has also been shown that mere repetition has a minimal cumulative impact on the specificity and precision of memory trace. For example, the phenomenon of "registration without learning" reveals that repetition need not be associated with an increase in the total number of features or details stored in a memory trace (Hintzman et al., 1992; Sheffert and Shiffrin, 2003; Kim et al., 2012). Thus, in contrast to judgments of frequency, which increase monotonically with repetition, the ability to discriminate a similar lure from a target item increases very little after the initial presentation (Malmberg et al., 2004), a finding that argues that the specificity of a mnemonic representation does not invariably increase with item repetition. When attention is directed in such a way as to encourage subjects to attend to features of a stimulus, however, then repetition has a beneficial impact on recollective memory. It is possible that in the present study, because of the absence of "similar lures," subjects fell into a retrieval mode that relied on familiarity judgments, setting the conditions for registration without learning to have occurred. As we have shown previously (Buchsbaum et al., 2011b), however, recency-related LIPC-AG effects are automatic, occurring even when subjects were not attending to the recently repeated item.

Thus, registration without learning phenomenon may explain the failure of repetition to increase activation in the LIPC-AG, especially under our preferred interpretation that this region indexes the degree of contextual specificity during mnemonic retrieval. It is useful here to observe that most definitions of recollection, and episodic memory more generally, state that it involves memory for what has happened at "particular places at particular times" (Tulving, 2002). However, in the case of memory judgments for an item that has been encountered multiple times within the experiment, then with respect to recollection of a repeated item, one must ask, "Which time?" That is, which particular repetition, which instance of experience, is recollected? On the other hand, indeed, is it some mnemonic amalgamation of the several repeated instances? We propose that in the case of recent repeated items (e.g., lag 1 or 2), the answer to this question is likely to be the last instance encountered. However, for longer lag trials (e.g., 16 and 32), where the temporal distance (Glenberg and Swanson, 1986; Brown et al., 2007) between successive repetitions is larger, the probability that the most recently encoded instance is recollected substantially decreases. Thus, the distinc- 
tion between lag and repetition on activity in the LIPC-AG may relate to the contextual specificity of the retrieved instance. Performance increases associated with repetition may be explained as a summation of several individually weak memory traces (Hintzman, 1988) that together contribute to a context-free familiarity signal, whereas lag effects may be due primarily to the predominance of a single strong memory trace that stands out against background signal distribution.

In summary, we have shown that repetition and recency, two factors that have large effects on memory performance, give rise to dissociable patterns of activation in the brain. Furthermore, we have shown that the LIPC-AG is sensitive to recency but not repetition, a finding that supports the hypothesis that this area indexes the degree of contextual specificity during memory retrieval.

\section{References}

Abdi H (2010) Barycentric discriminant analysis (BADIA). In: Encyclopedia of research design. Thousand Oaks: SAGE.

Abdi H, Williams LJ, Connolly AC, Gobbini MI, Dunlop JP, Haxby JV (2012) Multiple subject barycentric discriminant analysis (MUSUBADA): how to assign scans to categories without using spatial normalization. Comput Math Methods Med 2012:634165. CrossRef Medline

Avants BB, Epstein CL, Grossman M, Gee JC (2008) Symmetric diffeomorphic image registration with cross-correlation: evaluating automated labeling of elderly and neurodegenerative brain. Med Image Anal 12:26-41. CrossRef Medline

Berryhill ME (2012) Insights from neuropsychology: pinpointing the role of the posterior parietal cortex in episodic and working memory. Front Integr Neurosci 6:31. CrossRef Medline

Berryhill ME, Phuong L, Picasso L, Cabeza R, Olson IR (2007) Parietal lobe and episodic memory: bilateral damage causes impaired free recall of autobiographical memory. J Neurosci 27:14415-14423. CrossRef Medline

Brown GD, Neath I, Chater N (2007) A temporal ratio model of memory. Psychol Rev 114:539-576. CrossRef Medline

Buchsbaum BR, D’Esposito M (2009) Repetition suppression and reactivation in auditory-verbal short-term recognition memory. Cereb Cortex 19:1474-1485. CrossRef Medline

Buchsbaum BR, Padmanabhan A, Berman KF (2011a) The neural substrates of recognition memory for verbal information: spanning the divide between short-and long-term memory. J Cogn Neurosci 23:978-991. CrossRef Medline

Buchsbaum BR, Ye D, D'Esposito M (2011b) Recency effects in the inferior parietal lobe during verbal recognition memory. Front Hum Neurosci 5:59. CrossRef Medline

Buchsbaum BR, Lemire-Rodger S, Fang C, Abdi H (2012) The neural basis of vivid memory is patterned on perception. J Cogn Neurosci 24:18671883. CrossRef Medline

Ciaramelli E, Grady C, Levine B, Ween J, Moscovitch M (2010) Top-down and bottom-up attention to memory are dissociated in posterior parietal cortex: neuroimaging and neuropsychological evidence. J Neurosci 30: 4943-4956. CrossRef Medline

Cowan N (2001) The magical number 4 in short-term memory: a reconsideration of mental storage capacity. Behav Brain Sci 24:87-114; discussion 114-185. Medline

Cox RW (1996) AFNI: software for analysis and visualization of functional magnetic resonance neuroimages. Comput Biomed Res 29:162-173. CrossRef Medline

Dale AM, Fischl B, Sereno MI (1999) Cortical surface-based analysis: I. Segmentation and surface reconstruction. Neuroimage 9:179-194. CrossRef Medline

de Zubicaray GI, McMahon KL, Dennis S, Dunn JC (2011) Memory strength effects in fMRI studies: a matter of confidence. J Cogn Neurosci 23:2324-2335. CrossRef Medline

Dosenbach NU, Fair DA, Cohen AL, Schlaggar BL, Petersen SE (2008) A dual-networks architecture of top-down control. Trends Cogn Sci 12:99105. CrossRef Medline

Gardiner JM, Kaminska Z, Dixon M, Java RI (1996) Repetition of previously novel melodies sometimes increases both remember and know re- sponses in recognition memory. Psychon Bull Rev 3:366-371. CrossRef Medline

Glenberg AM, Swanson NG (1986) A temporal distinctiveness theory of recency and modality effects. J Exp Psychol Learn Mem Cogn 12:3-15. CrossRef Medline

Göthe K, Oberauer K (2008) The integration of familiarity and recollection information in short-term recognition: modeling speed-accuracy tradeoff functions. Psychol Res 72:289-303. CrossRef Medline

Greve A, Doidge AN, Evans CJ, Wilding EL (2010) Functional neuroanatomy supporting judgments of when events occurred. J Neurosci 30:70997104. CrossRef Medline

Henson RN, Rugg MD, Shallice T, Josephs O, Dolan RJ (1999) Recollection and familiarity in recognition memory: an event-related functional magnetic resonance imaging study. J Neurosci 19:3962-3972. Medline

Hintzman D (1988) Judgments of frequency and recognition memory in a multiple-trace memory model. Psychological Rev 95:528-551. CrossRef

Hintzman DL (2010) How does repetition affect memory? Evidence from judgments of recency. Mem Cognit 38:102-115. CrossRef Medline

Hintzman DL, Carre FA, Eskridge VL, Owens AM, Shaff SS, Sparks ME (1972) "Stroop" effect: input or output phenomenon? J Exp Psychol 95: 458-459. CrossRef Medline

Hintzman DL, Curran T, Oppy B (1992) Effects of similarity and repetition on memory: registration without learning? J Exp Psychol Learn Mem Cogn 18:667-680. CrossRef Medline

Hower KH, Wixted J, Berryhill ME, Olson IR (2014) Impaired perception of mnemonic oldness, but not mnemonic newness, after parietal lobe damage. Neuropsychologia 56:409-417. CrossRef Medline

Huijbers W, Pennartz CM, Daselaar SM (2010) Dissociating the "retrieval success" regions of the brain: effects of retrieval delay. Neuropsychologia 48:491-497. CrossRef Medline

Hutchinson JB, Uncapher MR, Wagner AD (2009a) Posterior parietal cortex and episodic retrieval: convergent and divergent effects of attention and memory. Learn Mem 16:343-356. CrossRef Medline

Johnson JD, Suzuki M, Rugg MD (2013) Recollection, familiarity, and content-sensitivity in lateral parietal cortex: a high-resolution fMRI study. Front Hum Neurosci 7:219. CrossRef Medline

Jones TC, Jacoby LL (2001) Feature and conjunction errors in recognition memory: evidence for dual-process theory. J Mem Lang 45:82-102. CrossRef

Kahn I, Davachi L, Wagner AD (2004) Functional-neuroanatomic correlates of recollection: implications for models of recognition memory. J Neurosci 24:4172-4180. CrossRef Medline

Kim H, Cabeza R (2009) Common and specific brain regions in high- versus low-confidence recognition memory. Brain Res 1282:103-113. CrossRef Medline

Kim K, Yi DJ, Raye CL, Johnson MK (2012) Negative effects of item repetition on source memory. Mem Cognit 40:889-901. CrossRef Medline

Kimura HM, Hirose S, Kunimatsu A, Chikazoe J, Jimura K, Watanabe T, Abe O, Ohtomo K, Miyashita Y, Konishi S (2010) Differential temporoparietal cortical networks that support relational and item-based recency judgments. Neuroimage 49:3474-3480. CrossRef Medline

Kuhl BA, Chun MM (2014) Successful remembering elicits event-specific activity patterns in lateral parietal cortex. J Neurosci 34:8051-8060. CrossRef Medline

Levy DA (2012) Towards an understanding of parietal mnemonic processes: some conceptual guideposts. Front Integr Neurosci 6:41. CrossRef Medline

Malmberg KJ, Holden JE, Shiffren RM (2004) Modeling the effects of repetitions, similarity, and normative word frequency on old-new recognition and judgments of frequency. J Exp Psychol Learn Mem Cogn 30:319-331. CrossRef Medline

Martin E (1968) Stimulus meaningfulness and paired-associate transfer: an encoding variability hypothesis. Psychol Rev 75:421-441. CrossRef Medline

McElree B, Dosher BA (1989) Serial position and set size in short-term memory: the time course of recognition. J Exp Psychol 118:346. CrossRef

McIntosh AR, Lobaugh NJ (2004) Partial least-squares analysis of neuroimaging data: applications and advances. Neuroimage 23:S250-263. CrossRef Medline

Melton AW (1970) The situation with respect to the spacing of repetitions and memory. J Verb Learn Verb Behav 9:596-606. CrossRef

Menon V, Uddin LQ (2010) Saliency, switching, attention and control: a 
network model of insula function. Brain Struct Funct 214:655-667. CrossRef Medline

Moritz S, Gläscher J, Sommer T, Büchel C, Braus DF (2006) Neural correlates of memory confidence. Neuroimage 33:1188-1193. CrossRef Medline

Nee DE, Jonides J (2008) Neural correlates of access to short-term memory. Proc Natl Acad Sci U S A 105:14228-14233. CrossRef Medline

Nee DE, Jonides J (2011) Dissociable contributions of prefrontal cortex and the hippocampus to short-term memory: evidence for a 3-state model of memory. Neuroimage 54:1540-1548. CrossRef Medline

Olson IR, Berryhill M (2009) Some surprising findings on the involvement of the parietal lobe in human memory. Neurobiol Learn Mem 91:155165. CrossRef Medline

Oztekin I, Davachi L, McElree B (2010) Are representations in working memory distinct from representations in long-term memory? Neural evidence in support of a single store. Psychol Sci 21:1123-1133. CrossRef Medline

Peterson LR, Johnson ST, Coatney R (1969) The effect of repeated occurrences on judgments of recency. J Verb Learn Verb Behav 8:591-596. CrossRef

Pinheiro JC, Bates DM (2000) Mixed-effects models in S and S-PLUS. New York: Springer.

Rissman J, Greely HT, Wagner AD (2010) Detecting individual memories through the neural decoding of memory states and past experience. Proc Natl Acad Sci U S A 107:9849-9854. CrossRef Medline

Sheffert SM, Shiffrin RM (2003) Auditory registration without learning. J Exp Psychol Learn Mem Cogn 29:10-21. CrossRef Medline

Simons JS, Peers PV, Hwang DY, Ally BA, Fletcher PC, Budson AE (2008) Is the parietal lobe necessary for recollection in humans? Neuropsychologia 46:1185-1191. CrossRef Medline

Tulving E (2002) Episodic memory: from mind to brain. Annu Rev Psychol 53:1-25. CrossRef Medline

Vilberg KL, Rugg MD (2007) Dissociation of the neural correlates of recognition memory according to familiarity, recollection, and amount of recollected information. Neuropsychologia 45:2216-2225. CrossRef Medline

Wagner AD, Shannon BJ, Kahn I, Buckner RL (2005) Parietal lobe contributions to episodic memory retrieval. Trends Cogn Sci 9:445-453. CrossRef Medline

Wells J (1974) Strength theory and judgments of recency and frequency. J Verb Learn Verb Behav 13:378-392. CrossRef

Wheeler ME, Buckner RL (2004) Functional-anatomic correlates of remembering and knowing. Neuroimage 21:1337-1349. CrossRef Medline

Wickelgren WA (1974) Single-trace fragility theory of memory dynamics. Mem Cognit 2:775-780. CrossRef Medline

Wilson MD (1988) The MRC psycholinguistic database: machine readable dictionary, version 2. Behav Res Method Instr Comput 20:6-11. CrossRef

Xue G, Dong Q, Chen C, Lu Z, Mumford JA, Poldrack RA (2010) Greater neural pattern similarity across repetitions is associated with better memory. Science 330:97-101. CrossRef Medline

Yassa MA, Stark CE (2008) Multiple signals of recognition memory in the medial temporal lobe. Hippocampus 18:945-954. CrossRef Medline

Yonelinas AP, Otten LJ, Shaw KN, Rugg MD (2005) Separating the brain regions involved in recollection and familiarity in recognition memory. J Neurosci 25:3002-3008. CrossRef Medline 